\title{
Effects of CCCP on the expression of GABARAPL2 in C6 glioma cells
}

\author{
Yoshimitsu Kiriyama, Airi Ozaki, Katsuhito Kino and Hiromi Nochi* \\ Tokushima Bunri University, Kagawa School of Pharmaceutical Sciences, Japan
}

\begin{abstract}
Astrocytes play an important role in the central nervous system. Ischemia, hypoxia, and traumatic injury cause mitochondrial damage in neurons and astrocytes. Damaged mitochondria are isolated and removed by macroautophagy (hereafter referred to as autophagy), which degrades cellular components with autophagosomes. Autophagy that removes mitochondria is called mitophagy. Phosphatidylethanolamine (PE)-conjugated microtubule-associated protein 1 light chain $3 \mathrm{~B}$ (LC3B) (LC3B-II) plays a crucial role in the formation of autophagosomes, and the conversion of unconjugated-LC3B (LC3B-I) to LC3B-II is the index of the induction of autophagy. Gamma-aminobutyric acid receptor-associated protein-like 2 (GABARAPL2) is the paralog of LC3B, and its function is not clear. In this study, we demonstrated that both the expression of total GABARAPL2 and the conversion of unconjugated-GABARAPL2 (GABARAPL2-I) to PE-conjugated GABARAPL2 (GABARAPL2-II) were upregulated by carbonyl cyanide 3-chlorophenylhydrazine (CCCP), which induces mitochondrial dysfunction and fragmentation. In contrast, CCCP upregulated the conversion of LC3B-I to LC3B-II, but it did not affect the expression of total LC3B. Thus, GABARAPL2 might function in the process of mitophagy during the formation of autophagosomes, and the expressions of GABARAPL2 and LC3B were regulated differently. The mechanism underlying the role of GABARAPL2 in mitophagy requires further investigations.
\end{abstract}

\section{Introduction}

Astrocytes function to form the blood-brain barrier, provide nutrients to neurons, retain the extracellular ion balance, surround synapses, and process information with neurons [1-4]. Ischemia, hypoxia and traumatic injury cause oxidative stress which results in mitochondrial damage [5-7]. This mitochondrial damage leads to the dysfunction and death of astrocytes [8,9]. Therefore, the isolation and removal of damaged mitochondria are necessary to maintain the function of astrocytes.

Macroautophagy (hereafter referred to as autophagy) is the bulk degradation process of cellular cytosolic components, such as defective proteins and damaged organelles [10]. The processes in autophagy include the stages of initiation, elongation and closure, and maturation [11-14]. In the process of autophagy, cellular cytosolic components are isolated by the autophagosome. The autophagosome is formed during the stage of elongation and closure, and the important proteins that form autophagosomes are the members of the microtubule-associated protein 1 light chain 3 (LC3) subfamily and gamma-aminobutyricacid-type-A receptor-associated protein (GABARAP) subfamily. In rats, the LC3 subfamily includes LC3A and LC3B, and the GABARAP subfamily includes GABARAP, GABARAP-like 1 (GABARAPL1), and GABARAP-like 2 (GABARAPL2, which is also known as GATE-16) [15-19]. Among the LC3 subfamily and the GABARAP subfamily, LC3B is most commonly used for studying autophagy. LC3B is catalyzed by ATG4 and is then conjugated to phosphatidylethanolamine (PE) by the ATG12-ATG5-ATG16 complex. LC3B-PE (LC3B-II) plays a crucial role in the formation of the autophagosome, and the conversion of unconjugated-LC3B (LC3B-I) to LC3B-II is the index of the induction of autophagy [18]. Although the physiological functions of the members of the LC3 subfamily, except for LC3B, and the GABARAP subfamily are not clear, the members of the LC3 subfamily and GABARAP subfamily are linked to PE, and they play a role in the formation of autophagosomes [16,20]. The degradation of cellular contents by autophagy occurs through both nonselective and selective pathways. The degradation of damaged mitochondria is considered as the selective autophagy (mitophagy) [21]. Nix, which is an outer mitochondrial membrane protein, binds LC3A, GABARAP, GABARAPL1, and GABARAPL2 but only weakly binds LC3B. Nix and GABARAPL1 are engaged in mitophagy by carbonyl cyanide 3-chlorophenylhydrazone (CCCP) [22]. However, the functions of GABARAPL2 in damaged mitochondria remain unclear.

In this study, rat C6 glioma cells, which are often used as a model for astrocytes, were used to observe the expression of the mRNA of all of the members of the LC3 subfamily and GABARAP subfamily. In addition, we examined the effects of CCCP, which causes mitochondrial damage, on the expression of GABARAPL2 in C6 glioma cells.

\section{Materials and methods}

\section{Materials}

CCCP and an anti-actin antibody were from Sigma (St. Louis, MO, USA). Anti-LC3B antibody and anti-GABARAPL2 (GATE-16) antibody was from MBL (Nagoya, Japan). Horseradish peroxidaselinked secondary antibodies were from Zymed Laboratories (South San Francisco, CA, USA). Mitotracker Red CMXRos Mitochondria Probe was purchased from Lonza Walkersville, Inc (Walkersville, MD, USA).

Correspondence to: Hiromi Nochi, Ph.D, Tokushima Bunri University, Kagawa School of Pharmaceutical Sciences, Shido 1314-1, Sanuki, Kagawa 769-2193, Japan; Tel: +81-87-899-7100; Fax: +81-87-894-0181; E-mail: nochi@kph.bunri-u.ac.jp

Key words: autophagy, mitophagy, LC3, GABARAPL2, GATE-16, GABARAP

Received: April 04, 2015; Accepted: April 24, 2015; Published: April 27, 2015 


\section{Cell culture}

Rat C6 glioma cells were obtained from JCRB cell bank (Osaka, Japan) and were maintained in DMEM supplemented with 10\% FBS, $100 \mathrm{units} / \mathrm{ml}$ penicillin, and $100 \mu \mathrm{g} / \mathrm{ml}$ streptomycin.

\section{Reverse transcription - PCR}

Total RNA isolated from cells was purified using RNeasy Mini Kits (Qiagen, Valencia, CA, USA). Reverse transcriptase reactions were performed on $1 \mu \mathrm{g}$ of RNA using a PrimeScript II 1st strand cDNA Synthesis Kit (Takara Bio, Otsu, Japan) in a $20 \mu$ l volume at $42^{\circ} \mathrm{C}$ for $60 \mathrm{~min}$. PCR was performed for 45 cycles using EmeraldAmp MAX PCR Master Mix (Takara Bio, Otsu, Japan) with the following primers: LC3A, 5'-ATGCCCTCCGACCGGCCTTTC- ${ }^{\prime}$ and 5'-TCAGAAGCCGAAGGTTTCT-3'; LC3B, 5' - ATGCCGTCCGAGAAGACCTTC-3' and 5'-TTACACAGCCAGTGCTGTCC-3'; Gabarap, 5'- ATGAAGTTCGTGTACAAAGA- $3^{\prime}$ and $5^{\prime}$-TCACAGACCGTAGACGCTTTC-3'; GabarapL1, 5'-ATGAAGTTCCAGTATAAAGA-3' and 5'-TCATTTCCCGTAGACACTTTC-3'; GabarapL2, 5'-ATGAAGTGGATGTTTAAGGA-3' and $5^{\prime}$-TCAGAAGCCAAAAGTGTTC- ${ }^{\prime}$.

All primer sets were selected to span intron(s) to obtain mRNAspecific PCR products and to amplify between $300 \mathrm{bp}$ and $400 \mathrm{bp}$.

\section{Western blot analysis}

C6 glioma cells treated with vehicle or CCCP were harvested and lysed in modified RIPA buffer (50 mM Tris- $\mathrm{HCl}, \mathrm{pH} 7.4,1 \%$ NP-40, $0.25 \%$ sodium deoxycholate, $150 \mathrm{mM} \mathrm{NaCl}, 1 \mathrm{mM}$ EDTA, $2 \mathrm{mM}$ diisopropylfluorophosphate, $10 \mu \mathrm{g} / \mathrm{mL}$ of leupeptin, $10 \mu \mathrm{g} / \mathrm{mL}$ of aprotinin, and $10 \mu \mathrm{g} / \mathrm{mL}$ of pepstatin) on ice for $20 \mathrm{~min}$. Cell lysates were sonicated and centrifuged at $12,000 \times \mathrm{g}$ at $4^{\circ} \mathrm{C}$ for $10 \mathrm{~min}$. Proteins in the supernatants were electrophoresed in sodium dodecyl sulfatepolyacrylamide gel electrophoresis (SDS-PAGE) and transferred to Immobilon $\mathrm{P}$ membranes (Millipore, Billerica, MA). The membranes were blocked with $1 \%$ skim milk in $0.1 \%$ Tween $20 / \mathrm{PBS}$ (PBS-T) and incubated with primary antibodies (dilutions based on manufacturer's recommendations). The membranes were washed three times in

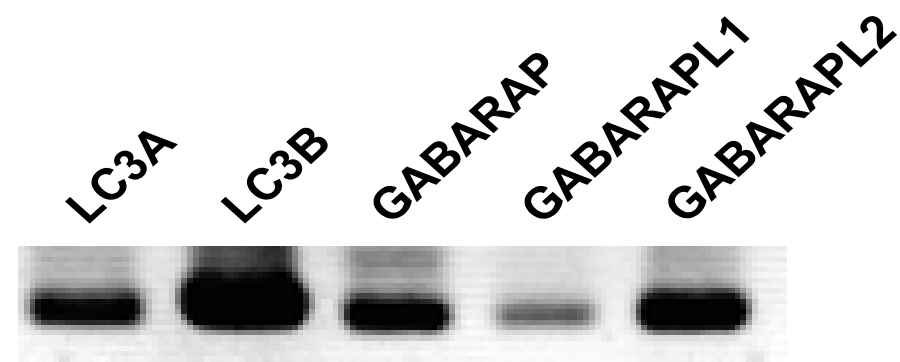

Figure 1. The expression of mRNAs for the members of LC3 subfamily and GABARAP subfamily in C6 glioma cells. Total RNA was isolated from C6 glioma cells and LC3A LC3B, GABARAP, GABARAPL1, and GABARAPL2 mRNA expression were detected by RT-PCR for 45 cycles. All primer sets spanned more than one intron of the target gene and were designed to amplify between $300 \mathrm{bp}$ and $400 \mathrm{bp}$.
A
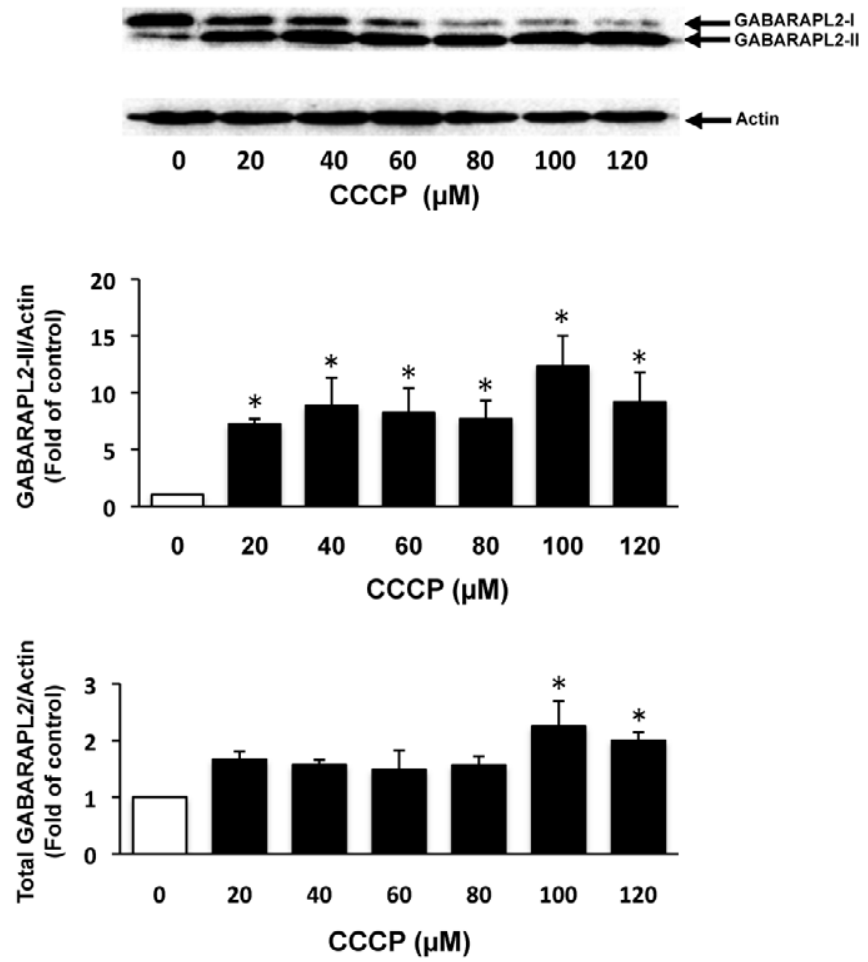

B
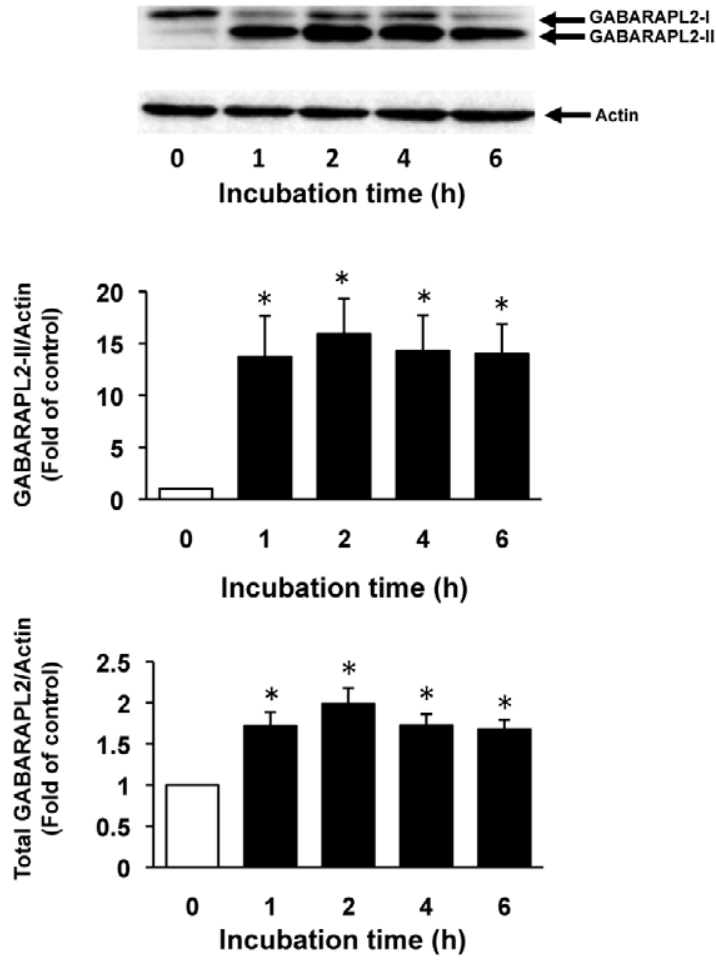

Figure 2. GABARAPL2 expression by CCCP in C6 glioma cells. (A) C6 glioma cells were treated with the indicated concentrations of CCCP for 4h. B. C6 glioma cells were treated with $120 \mu \mathrm{M}$ of CCCP for the indicated times. Cell lysates were subjected to a western blot analysis with an anti-GABARAPL2 antibody. The expression levels of GABARAPL2 were compared with those of actin, which was used as an internal control. The results are the ratios of the treated to control levels (means \pm SEM, $n=3$ ). $p$ values $<0.05$ were considered statistically significant. 
PBS-T and incubated for $1 \mathrm{~h}$ with a horseradish peroxidase-conjugated secondary antibody. Immunoreactive proteins were detected with ImmunoStar LD (Wako Pure Chemical Industries, Osaka, Japan).

\section{Mitochondria staining}

Rat C6 glioma cells were cultured on 35-mm glass-bottomed dishes (Iwaki, Shizuoka, Japan). The cells were treated with CCCP for $4 \mathrm{~h}$. Mitotracker Red CMXRos Mitochondria Probe (final concentrations of $50 \mathrm{nM}$ ) was added at 30 minutes before the end of incubation. Images were acquired using a Leica DMI 6000 B microscope with AF6000 software (Leica, Wetzlar, Germany).

\section{Statistical analysis}

Results of multiple observations are presented as means \pm SEM. One-way analysis of variance (ANOVA) followed by the Dunnett's test was used for multiple comparisons. $\mathrm{P}<0.05$ was considered significant.

\section{Results}

The levels of mRNA expression of the members of the LC3 subfamily and GABARAP subfamily in C6 glioma cells were assessed by RT-PCR (Figure 1). The mRNAs of all of the members of the LC3 subfamily and GABARAP subfamily were expressed in C6 glioma cells. The mRNA of LC3B was expressed most abundantly, and the mRNAs of LC3A, GABARAP, and GABARAPL2 were moderately expressed. The mRNA of GABARAPL1 was slightly expressed. We examined the effects of CCCP on the expression of GABARAPL2-II and total GABARAPL2. GABARAPL2-II, which is the PE-conjugated form of GABARAPL2, can bind autophagosomes. Thus, the levels of GABARAPL2-II are considered to reflect the activity of GABARAPL2. GABARAPL2-I, which is the unconjugated form, migrates slower than GABARAPL2-II on SDS-PAGE [16]. GABARAPL2-II expression levels were increased seven times compared to that of the control in response to $20 \mu \mathrm{M}$ of CCCP, and the increase in GABARAPL2II expression reached a maximum (12 times that of the control) in response to $100 \mu \mathrm{M}$ of CCCP. Total GABARAPL2 expression levels increased to twice that of the control in response to $100 \mu \mathrm{M}$ of CCCP, and total GABARAPL2 expression in response to $120 \mu \mathrm{M}$ of CCCP remained at this level (Figure $2 \mathrm{~A}$ ). In addition, we investigated the effects of $120 \mu \mathrm{M}$ of CCCP on the expression of GABARAPL2-II and total GABARAPL2 at various time points up to $6 \mathrm{~h}$. GABARAPL2-II was increased to 13 times that of the control in response to $120 \mu \mathrm{M}$ of CCCP at $1 \mathrm{~h}$, after which the expression level of GABARAPL2-II remained at this level. Total GABARAPL2 was increased about twice that of control in response to $120 \mu \mathrm{M}$ of CCCP at $1 \mathrm{~h}$, after which the GABARAPL2 expression remained at this level.

The effects of $120 \mu \mathrm{M}$ of CCCP on the expression of LC3 were examined at various time points up to $6 \mathrm{~h}$ (Figure $3 \mathrm{~A}$ ) because the conversion of LC3 from LC3-I to LC3-II reflects the induction of autophagy and is a key step in the forming of autophagosomes. Similar to the results of GABARAPL2, LC3B-II expression was increased about ten times that of the control in response to $120 \mu \mathrm{M}$ of CCCP at $1 \mathrm{~h}$, after which it remained at this level. However, total LC3B expression levels were not affected by $120 \mu \mathrm{M}$ of CCCP (Figure 3A). The fragmentation of mitochondria precedes mitophagy [23]. We confirmed that the reticular mitochondrial network was fragmented by $120 \mu \mathrm{M}$ of CCCP (Figure 3B). These results indicated that mitochondrial dysfunction correlated with the expression and activity of GABARAPL2.

\section{A}

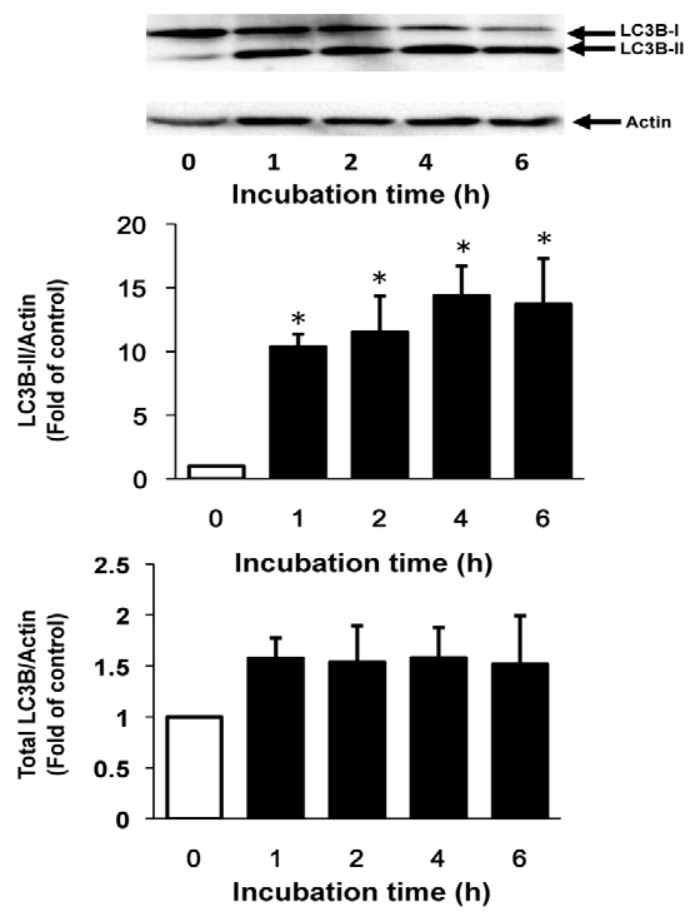

B
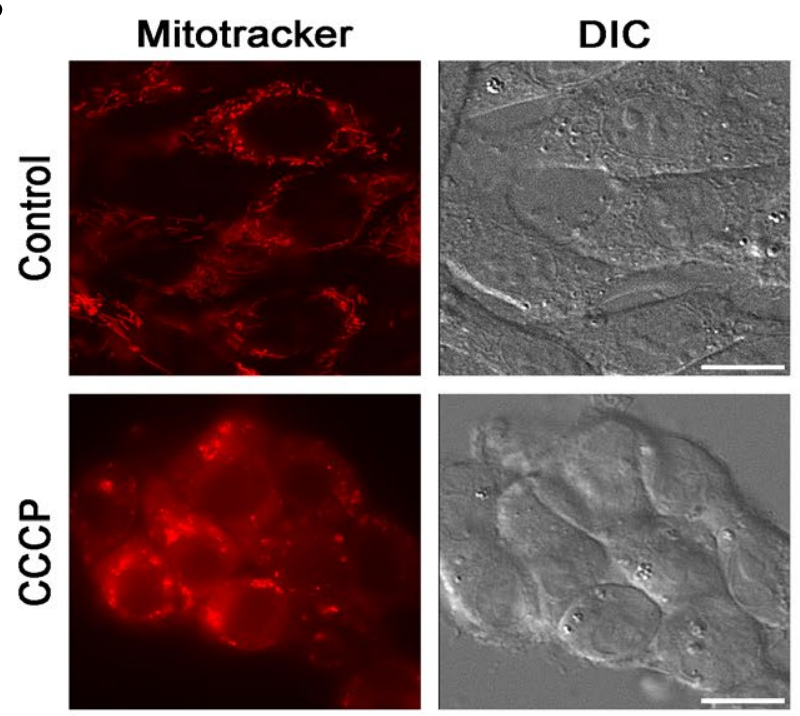

Figure 3. LC3B expression and the change in mitochondrial morphology in response to CCCP. (A) $\mathrm{C} 6$ glioma cells were treated with $120 \mu \mathrm{M}$ of CCCP for the indicated times. Cell lysates were subjected to a western blot analysis with an anti-LC3B antibody. The expression levels of LC3B were compared with those of actin, which was used as an internal control. Results are the ratios of treated to control levels (means $\pm \mathrm{SEM}, \mathrm{n}=3$ ). $p$ values $<0.05$ were considered statistically significant. (B) $\mathrm{C} 6$ glioma cells were treated with vehicle or $120 \mu \mathrm{M}$ of CCCP for $4 \mathrm{~h}$, and the morphology of mitochondria was visualized with mitotracker. Corresponding DIC images are shown. Scale bars, $10 \mu \mathrm{m}$. 


\section{Discussion}

The segregation and exclusion of damaged mitochondria are crucial to maintain the function of astrocytes. Damaged mitochondria are sequestered and eliminated by mitophagy, which is accompanied by the formation of autophagosomes. LC3B, which is a member of the LC3 subfamily and GABARAP subfamily, is a well-characterized protein that is involved in the formation of autophagosomes. However, the functions of the other members of the LC3 subfamily and GABARAP subfamily have not been completely elucidated. Although the physiological functions of GABARAPL2 in mitophagy are not well understood, it has been shown that GABARAPL2 is linked to phospholipids in the same manner as LC3 phospholipidation and may play a role in autophagy [16]. Moreover, GABARAPL2 binds to an ubiquitin-binding protein, p62 [24], and to a mitochondrion-localized protein, Nix [22]. Both p62 and Nix are involved in mitophagy that is induced by CCCP. In CCCPinduced mitophagy, voltage-dependent anion channels (VDACs), which are on the surface of mitochondria, are ubiquitinated by PINK1 and Parkin, and p62 then binds ubiquitinated-VDACs. LC3B carries p62 with ubiquitinated-VDACs to the autophagosome $[21,25,26]$. On the other hand, Nix interacts with GABARAPL1, and GABARAPL1 then brings the mitochondria to the autophagosome during the CCCP-induced mitophagy [22]. CCCP is the uncoupler that induces the fragmentation of mitochondria, which precedes mitophagy [23]. Therefore, we examined the effects of CCCP on the activation and expression of GABARAPL2. CCCP induced the upregulation of both GABARAPL2-II and total GABARAPL2. In contrast, the treatment of CCCP resulted in LC3-II upregulation, which is the indicator of the formation of the autophagosome, while CCCP did not affect the levels of expression of total LC3B. Recent studies have shown that LC3B plays a role in the early stage of autophagy (elongation of the phagophore membrane) and that GABARAPL2 is necessary for the late stage of autophagy (autophagosome closure) [20]. Therefore, GABARAPL2 may function in autophagosome closure during mitophagy, and each member of the LC3 subfamily and GABARAP subfamily may have distinct roles in the process of autophagy.

In conclusion, we demonstrated that both GABARAPL2-II and total GABARAPL2 were upregulated by CCCP in C6 glioma cells. GABARAPL2 possibly functions in the segregation of damaged mitochondria that occurs to protect against the dysfunction and death of astrocytes. The mechanism involving GABARAPL2 in the clearance of damaged mitochondria in astrocytes and the functions of GABARAPL2 require further investigations.

\section{References}

1. Allaman I, Bélanger M, Magistretti PJ (2011) Astrocyte-neuron metabolic relationships: for better and for worse. Trends Neurosci 34: 76-87. [Crossref]

2. Ballabh P, Braun A, Nedergaard M (2004) The blood-brain barrier: an overview: structure, regulation, and clinical implications. Neurobiol Dis 16: 1-13. [Crossref]

3. Parpura V, Heneka MT, Montana V, Oliet SH, Schousboe A, et al. (2012) Glial cells in (patho) physiology. J Neurochem 121: 4-27. [Crossref]

4. Stobart JL, Anderson CM (2013) Multifunctional role of astrocytes as gatekeepers of neuronal energy supply. Front Cell Neurosci 7: 38. [Crossref]

5. Chen H, Yoshioka H, Kim GS, Jung JE, Okami N, et al. (2011) Oxidative stress in ischemic brain damage: mechanisms of cell death and potential molecular targets for neuroprotection. Antioxid Redox Signal 14: 1505-1517. [Crossref]

6. Kochanek PM, Jackson TC, Ferguson NM, Carlson SW, Simon DW1, et al. (2015) Emerging therapies in traumatic brain injury. Semin Neurol 35: 83-100. [Crossref]

7. Sena LA, Li S, Jairaman A, Prakriya M, Ezponda T, et al. (2013) Mitochondria are required for antigen-specific $\mathrm{T}$ cell activation through reactive oxygen species signaling. Immunity 38: 225-236. [Crossref]
8. Cabezas R, El-Bachá RS, González J, Barreto GE (2012) Mitochondrial functions in astrocytes: neuroprotective implications from oxidative damage by rotenone. Neurosci Res 74: 80-90. [Crossref]

9. Takuma K, Baba A, Matsuda T (2004) Astrocyte apoptosis: implications for neuroprotection. Prog Neurobiol 72: 111-127. [Crossref]

10. Kroemer G, Marino G, Levine B (2010) Autophagy and the integrated stress response. Mol Cell 40: 280-293. [Crossref]

11. Feng Y, He D, Yao Z, Klionsky DJ (2014) The machinery of macroautophagy. Cell Res 24: 24-41. [Crossref]

12. Kiriyama Y, Kino K, Nochi H (2015) Autophagy and amino acids with their metabolites. Integrative Food, Nutrition and Metabolism 2: 151-155.

13. Mizushima N, Komatsu M (2011) Autophagy: renovation of cells and tissues. Cell 147: 728-741. [Crossref]

14. Shen HM, Mizushima N2 (2014) At the end of the autophagic road: an emerging understanding of lysosomal functions in autophagy. Trends Biochem Sci 39: 61-71. [Crossref]

15. Kabeya Y, Mizushima N, Ueno T, Yamamoto A, Kirisako T, et al. (2000) LC3, a mammalian homologue of yeast Apg8p, is localized in autophagosome membranes after processing. EMBOJ 19: 5720-5728. [Crossref]

16. Kabeya Y, Mizushima N, Yamamoto A, Oshitani-Okamoto S, Ohsumi Y, et al. (2004) LC3, GABARAP and GATE16 localize to autophagosomal membrane depending on form-II formation. J Cell Sci 117: 2805-2812. [Crossref]

17. Tanida I, Komatsu M, Ueno T, Kominami E (2003) GATE-16 and GABARAP are authentic modifiers mediated by Apg7 and Apg3. Biochem Biophys Res Commun 300: 637-644. [Crossref]

18. Wild P, McEwan DG, Dikic I (2014) The LC3 interactome at a glance. J Cell Sci 127 3-9. [Crossref]

19. Xin Y, Yu L, Chen Z, Zheng L, Fu Q, et al. (2001) Cloning, expression patterns, and chromosome localization of three human and two mouse homologues of GABA(A) receptor-associated protein. Genomics 74: 408-413. [Crossref]

20. Weidberg H, Shvets E, Shpilka T, Shimron F, Shinder V, et al. (2010) LC3 and GATE16/GABARAP subfamilies are both essential yet act differently in autophagosome biogenesis. EMBOJ 29: 1792-1802. [Crossref]

21. Youle RJ, Narendra DP (2011) Mechanisms of mitophagy. Nat Rev Mol Cell Biol 12: 9-14. [Crossref]

22. Novak I, Kirkin V, McEwan DG, Zhang J, Wild P, et al. (2010) Nix is a selective autophagy receptor for mitochondrial clearance. EMBO Rep 11: 45-51. [Crossref]

23. Gomes LC, Scorrano L (2013) Mitochondrial morphology in mitophagy and macroautophagy. Biochim Biophys Acta 1833: 205-212. [Crossref]

24. Pankiv S, Clausen TH, Lamark T, Brech A, Bruun JA, et al. (2007) p62/SQSTM1 binds directly to Atg8/LC3 to facilitate degradation of ubiquitinated protein aggregates by autophagy. J Biol Chem 282: 24131-24145. [Crossref]

25. Geisler S, Holmström KM, Skujat D, Fiesel FC, Rothfuss OC, et al. (2010) PINK1/ Parkin-mediated mitophagy is dependent on VDAC1 and p62/SQSTM1. Nat Cell Biol 12: 119-131. [Crossref]

26. Johansen T, Lamark T (2011) Selective autophagy mediated by autophagic adapter proteins. Autophagy 7: 279-296. [Crossref]

Copyright: (C2015 Kiriyama Y. This is an open-access article distributed under the terms of the Creative Commons Attribution License, which permits unrestricted use, distribution, and reproduction in any medium, provided the original author and source are credited. 